\title{
Reliability, Standardization, and Validation of Optical Fiber Sensors
}

\author{
Rolf Brönnimann, Marcel Held, Philipp M. Nellen, \\ Empa, Materials Science and Technology, Laboratory Electronics/Metrology \\ CH-8600 Dübendorf, Switzerland, T. +41 1823 48 82, F: +41 182340 54, rolf.broennimann@empa.ch
}

\begin{abstract}
For wider acceptance of optical fiber sensors standardized products are needed with well understood properties and validated performance characteristics. Reliability, availability, maintainability, validation, and standardization of fiber sensors are discussed.
\end{abstract}

OCIS codes: 060.2370

\section{Introduction}

Optical fiber sensors have well known advantages like low weight, flexibility, smallness, electromagnetic immunity, point and distributed sensing, but they have become common only in certain niche markets, e.g. fiber gyroscopes or oil field sensors. For wider acceptance standardized products are needed with well understood properties and with validated performance characteristics [1-4].

From the operator's point of view, monitoring a structure should allow making decisions concerning its condition, e.g. is it operational, does it need maintenance, remaining lifetime, etc. Many steps are required from a functional sensor towards a reliable monitoring system. It is impracticable to equip a structure with sensors in a way that its condition can be completely assessed. Therefore, at the beginning of the development of a monitoring system, one has to determine the essential parameters to be measured. Typically, the measurements are rather indirect, e. g. strain is measured which is related by Young's modulus and cross section to the actual parameter of interest, the load on a cable. It is assumed that the material is homogeneous and the cross section is uniformly loaded. Models are always used to find and support the conclusions, being it for the measurement results, the data analysis, or for reliability consideration of the measurement devices.

The components of a measurement system have to be selected with respect to their ability to deliver desired quantities as accurate as necessary without being disturbed by environmental or other influences. These quantities calculated from the sensor measurements are always based on models of the structure. Important criteria for the selection of components are reliability, availability on the market, price, familiarity, uniformity of the measurement system, standards, codes etc. The selection is usually the result of an optimization process.

There are many steps to be considered starting from the structure to final results that allow decisions (see Fig. 1). First, the important measurands, as load, displacement, humidity, temperature etc., have to be selected. Then appropriate transducing mechanisms to the sensors have to be found (fiber sensors clamped, glued, embedded, ...). As an example strain has to be transferred to an optical fiber containing a Bragg grating (FBG). The bond has to be sufficiently stable for the measurement range over the lifetime (typically, civil engineering structures are constructed for several decades) with all the anticipated environmental influences as temperature, humidity, pressure etc. The sensor will be still sensitive to undesired influences to some degree even if it is properly installed. Compensation schemes have to be applied either in the sensor itself or in the data evaluation. The sensor is then connected to an interrogation system by a feed line whose influence varies depending on the detection scheme. The feed line is also a source of failure (aging, damage). The interrogation system has two main parts, i.e. opto-electronics to read out the sensor and software algorithms to convert the raw data into significant values as well as time series. The values are stored in a data base where they are available for further analysis. Based on the analysis decisions could be made according to predefined criteria like overload, stress release, and others.

The required availability of a monitoring system depends on its relevance, the lifetime and also the required probability that anticipated events will be detected. The relevance or the purpose of a system can be performance verification of some elements of a structure, early identification of damage of structures or detection of security relevant changes. Depending on these the system can be short living or has to exceed the lifetime of the structure. Also the required detection probability depends on the application. A comparably low probability is acceptable, if early detection just helps to reduce cost. In other cases a high level of detection probability is necessary which 


\section{ThD1.pdf}

requires that redundancies in the sensor system have to be built in. Of course these requirements have a strong impact on cost.

\section{RAM: Reliability, Availability, Maintainability}

A high availability of a monitoring system over a prolonged time span can become very demanding and has to be defined before commissioning. First, the terms reliability, availability and maintainability have to be clarified. A common definition is [5]:

- $\quad$ Reliability: Probability that an item will perform its required function under given conditions for a stated time interval.

- Availability: Probability that an item will perform its required function under given conditions at a stated time.

- Maintainability: Ability of an item to be retained or restored to a specific state.

In addition to reliability, availability includes the maintainability of a system. Whereas the reliability function decays to zero with time, the availability can stay on a high level, if a system can be maintained. However, under certain conditions, the optical fiber sensors cannot be repaired (not accessible, embedded).

Reasons for an item not to perform its required function can be permanent failure or change of sensitivity or accuracy. Causes are aging, improper use or installation, or extreme events as temperature excursions, overload and chemical attack. Therefore, two different types of maintenance can be distinguished, i.e. on one hand repair, replacement or update of items and software and on the other hand performance verification and recalibration.

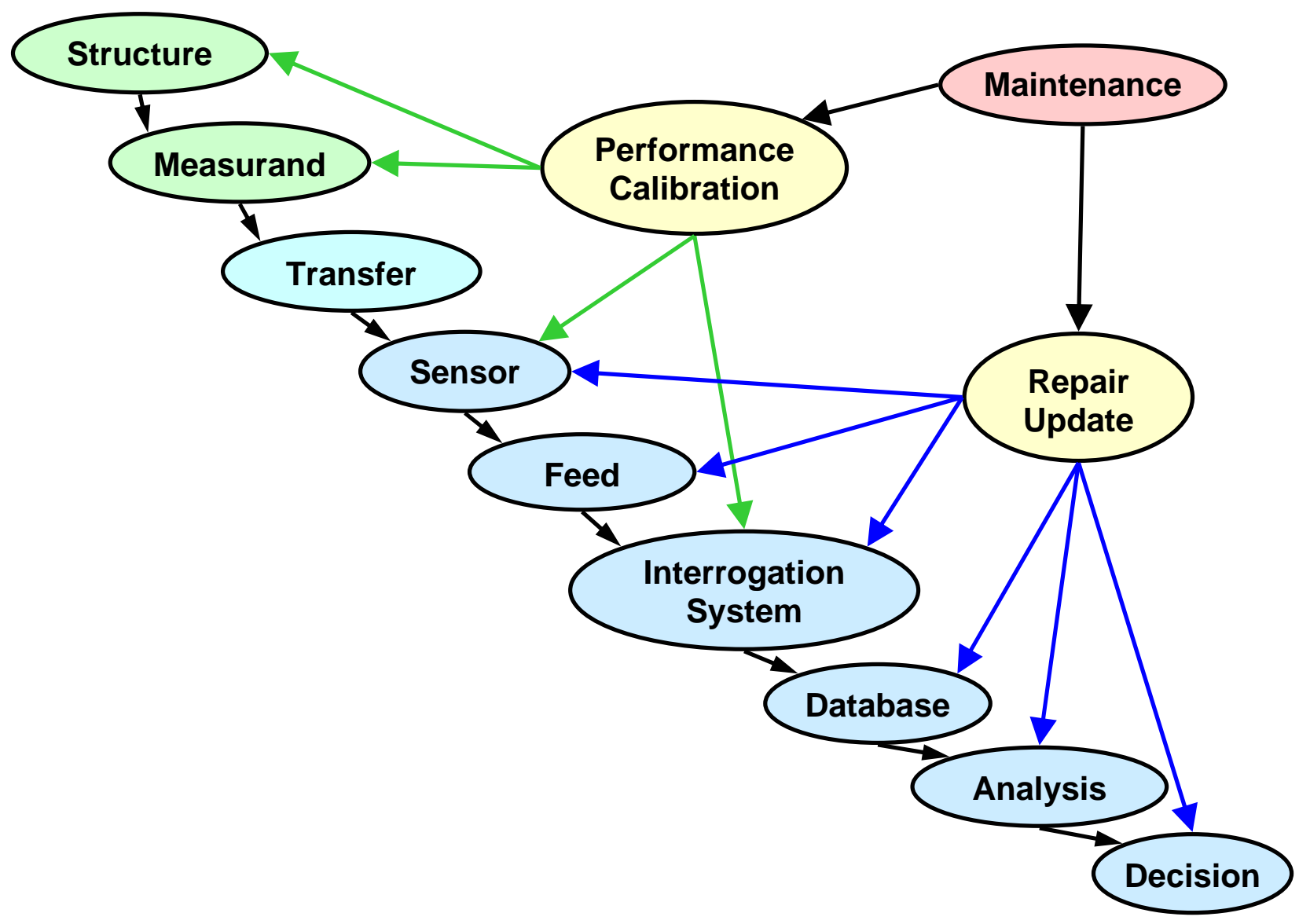

Fig. 1: Maintainability of a monitoring system 


\section{ThD1.pdf}

Performance verification or calibration applies for the elements "Structure" to "Interrogation System" in Fig. 1. The calibration of the interrogation system is usually possible since it is easily accessible. Sometimes it is possible to calibrate the complete chain by loading the structure in a defined way where the expected response is known. However, for structures like power dams or bridges this is impracticable. One can try to directly influence the measurand or determine it by other means. For example, the cables of a stayed cable bridge equipped with FBG can be calibrated with the same hydraulic press which is used to pretension the cables during construction. And finally, provisions can be built in to influence the sensor directly.

Repair, replacement or update can be easily done for the elements "Interrogation System" to "Decision" for optical fiber sensors. It depends on the installation if it is also possible for the sensors and the feed line. Embedded sensors are usually not replaceable and present the weak point of a monitoring system. Therefore comprehensive studies have to be made [4,6,and 7] to prove if an acceptable availability for long term monitoring can be achieved. Analysis of faults, estimation of their probabilities and sensitivity analysis help to identify critical parts and the improvement of which parts has most effect on lifetime. If improvements are not possible or costly also redundancies can be built in. It is not practical to perform such studies for each application. Therefore standardized validation procedures are needed.

\section{Fault tree}

Important is the understanding of the failure modes of sensors, their probability and means to improve reliability. A fault tree is a systematic hierarchical decomposition into events which lead to failure of an item. Fig. 2 shows a fault tree diagram for fiber Bragg grating (FBG) sensors used for cable monitoring. At first, sensor failure is dissected into the failures "insufficient light" and "insufficient accuracy". Each failure mode is then further analyzed. By weighting the importance of the events, understanding the mechanism that lead to the specific failure helps to identify where the system has to be improved. A more detailed but also more costly investigation can be made with FME(C)A (fault modes, effects, (and criticality) analysis) [5]. Examples of extensive investigations of various faults and validation of FBG are found elsewhere [e.g., 4, 9]. Creep of the fiber in the adhesive bond and break of FBG or fiber during operation caused by improper embedding, were found to be the most probable and thus critical failures. In addition to redundancies, provision for self diagnosis [4] can be built in which improves the confidence in the measured data significantly.

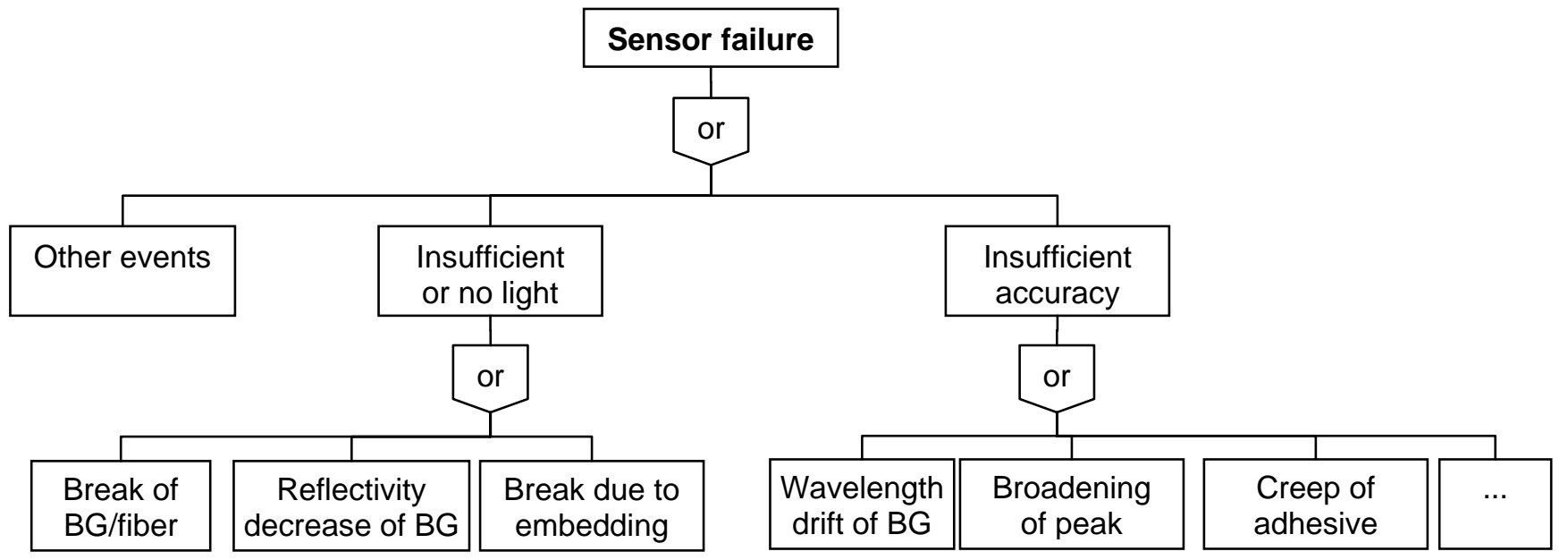

Fig. 2: Fault tree diagram for a FBG sensor system.

\section{Sensitivity analysis}

Sensitivity analysis is used to identify weak elements in a system and where improvement is most rewarding. The improvement can be a more reliable sensor design, provisions for maintenance and redundancies. One possibility of such analysis is the systematic variation of failure probabilities of the elements. A reliability block diagrams (RBD) 


\section{ThD1.pdf}

helps structuring the analysis. Fig. 3 (left) shows the RBD of the sensing system for a prestressing cable consisting of CFRP wires. Four wires were equipped with two to three FBG. They have been directly embedded during the pultrusion process in the middle of the CFRP wires [8]. The FBG named $A_{i}, B$, and $C$ are inside the anchor head, $F_{i j}$ are in the free span and $T_{i j}$ are for temperature measurement and compensation. The block diagram corresponds to a requirement where a FBG is available at least at each position inside the anchor head, one in the free span and one for compensation. From field data the MTTF (mean time to failure) of the various FBG has been estimated. The reliability can be evaluated either by straightforward calculations or by Monte-Carlo simulations.

The sensitivity analysis (Fig. 3 right) shows how the MTTF changes when the MTTF of one individual element is varied. It can be seen, that the dependence on elements B and C is the strongest and that these elements should be improved first.
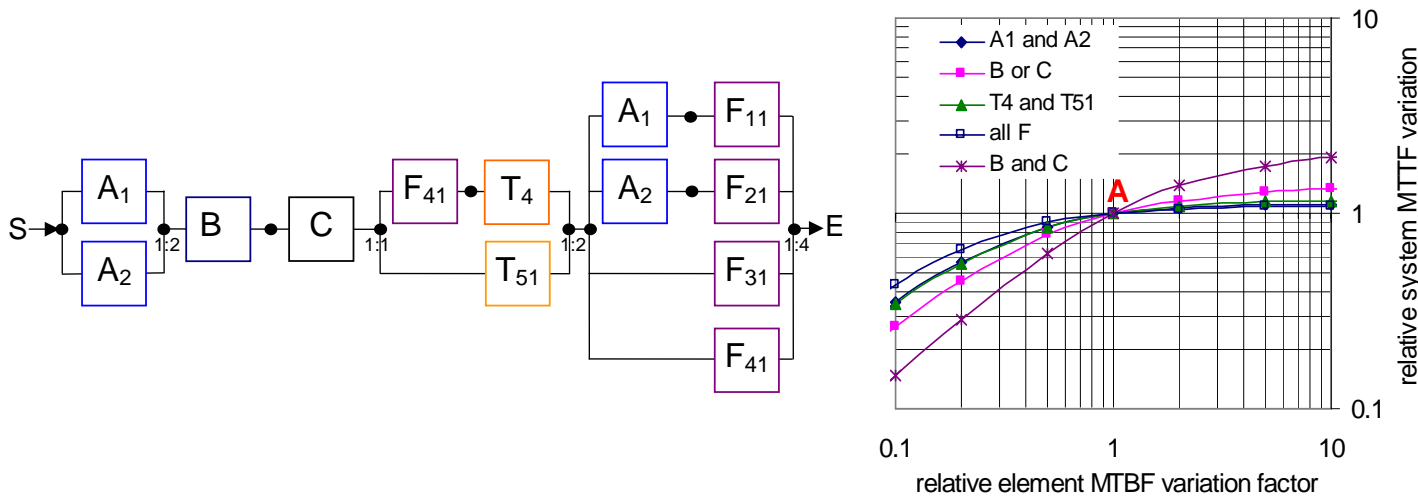

Fig. 3: Reliability block diagram for a CFRP cable with embedded FBG (left) and sensitivity analysis of the system (right)

\section{Concluding remarks}

It is recognized that for wider acceptance of fiber optical sensors standardized products are needed with well understood properties and with validated performance characteristics. Several pre-normative working groups in Europe, America and Asia are preparing first drafts as for example ISIS, COST 270/299, RILEM and others. Some recommendations that are used in telecom can be used but many problems particular to fiber optical sensing have to be addressed individually and guidelines still have to be established.

\section{References}

[1] Wolfgang R. Habel, Guidelines and standards for fiber optic sensors: Quo vadis?, SPIE 6167, 339-347, 2006

[2] B. Culshaw, W. Habel, Fibre Sensing: Specifying components and systems, NIST special publication 1024, Technical Digest: Symposium on optical fiber mesurements, 179-90, 2004

[3] F. Berghmans, Reliability of components for fiber optic sensors, SPIE 5758, 417-26, 2005

[4] Rolf Brönnimann, Marcel Held, Philipp M. Nellen, Reliability, availability, and maintainability considerations for fiber optical sensor applications, SPIE 6167, 348-362, 2006

[5] A.Birolini: Reliability engineering, Springer ISBN 3-540-66385-1, 2004

[6]. W. Griffioen, T. Breuls, G. Cocito, S. Dodd, G. Ferri, P. Hasløv, L. Oksanene, D. Stockton, T. Svensson, COST 218 evaluation of optical fibre lifetime models, SPIE 1791, 190-201, 1992

[7] M. J. Matthewson, Optical fiber reliability models, in Fiber optics reliability and testing, SPIE Conf. Proc., D. K. Paul, Ed., 1994

[8] R. Brönnimann, P. M. Nellen, U. Sennhauser, Reliability monitoring of CFRP structural elements in bridges with fiber optical Bragg grating sensors, J. Intelligent Material Systems and Structures, 10(4), 322-9, 1999

[9] Nellen P.M., Mauron P., Frank A., Sennhauser U., Bohnert K., Pequignot P., Bodor P., Brändle H. Reliability of fiber Bragg grating based sensors for downhole applications, Sensors and Actuators A 103 (2003) 364 - 376; .2003 